Journal of Applied Fluid Mechanics, Vol. 2, No. 1, pp. 23-28, 2009.

Available online at www.jafmonline.net, ISSN 1735-3572, EISSN 1735-3645.

DOI: $10.36884 / \mathrm{jafm} .2 .01 .11852$

\title{
Effects of Varying Viscosity and Thermal Conductivity on Steady Free Convective Flow and Heat Transfer Along an Isothermal Vertical Plate in the Presence of Heat Sink
}

\author{
N. C. Mahanti ${ }^{1}$ and P. Gaur ${ }^{2}$ \\ ${ }^{1}$ Department of Applied Mathematics, Birla Institute of Technology, Mesra, Ranchi-835215, India. \\ ${ }^{2}$ Department of Computer Science, Birla Institute of Technology, Mesra, Ranchi-835215, India. \\ Email: ncmahanti@rediffmail.com,pramodgaur82@gmail.com
}

(Received December 27, 2007; accepted April 27, 2008)

\begin{abstract}
Aim of the paper is to investigate the effects of linearly varying viscosity and thermal conductivity on steady free convective flow of a viscous incompressible fluid along an isothermal vertical plate in the presence of heat sink. The governing equations of continuity, momentum and energy are transformed into coupled and nonlinear ordinary differential equations using similarity transformation and then solved using Runge-Kutta fourth order method with shooting technique. The velocity and temperature distributions are discussed numerically and presented through graphs. Skin-friction coefficient and Nusselt number at the plate are derived, discussed numerically and their numerical values for various values of physical parameters are presented through Tables.
\end{abstract}

Keywords: Steady, free convection, variable viscosity, variable thermal conductivity, heat sink, skin-friction coefficient, Nusselt number.

$\begin{array}{ll}g & \text { Acceleration due to gravity of the Earth } \\ x, y & \text { cartesian coordinates } \\ f & \text { dimensionless velocity function } \\ G r & \text { Grashof number }\left\{=g \beta\left(T_{w}-T_{\infty}\right) x^{3} / v^{2}\right\} \\ N u & \text { Nusselt number } \\ P r & \text { Prandtl number }\left(=\mu C_{p} / \kappa\right) \\ C_{f} & \text { skin-friction coefficient } \\ C_{p} & \text { specific heat at constant pressure } \\ T & \text { temperature of the fluid } \\ T_{w} & \text { temperature of the plate } \\ T_{\infty} & \text { temperature of fluid far from plate } \\ u, v & \text { velocity components along } x \text { - and } y- \\ \beta & \text { directions } \\ \beta & \text { coefficient of thermal expansion }\end{array}$

\section{INTRODUCTION}

The natural convection process in the presence of heat source/sink is present in various physical phenomena such as fire engineering, combustion modelling, nuclear energy, heat exchangers, petroleum reservoir etc. Liquid metals having low Prandtl number (because of very large thermal conductivity) are generally used as coolants and have

$\begin{array}{ll}\eta & \text { similarity variable } \\ \varepsilon & \text { thermal conductivity parameter } \\ \gamma & \text { viscosity parameter } \\ \theta & \text { dimensionless temperature } \\ & \left\{=\left(T-T_{\infty}\right) /\left(T_{w}-T_{\infty}\right)\right\} \\ \mu^{*} & \text { variable viscosity } \\ \mu & \text { coefficient of viscosity } \\ \kappa^{*} & \text { variable thermal conductivity } \\ \kappa & \text { thermal conductivity } \\ v & \text { kinematic viscosity }(=\mu / \rho) \\ \rho & \text { fluid density } \\ , & \text { differentiation with respect to } \eta\end{array}$

applications in manufacturing processes such as the cooling of the metallic plate, nuclear reactor etc. Liquid metal has ability to transport heat even if small temperature difference exists between the surface and fluid. For this reason liquid metal is used as coolant in nuclear reactor to transfer waste heat from the core region. Ideally, it is expected that coolant should never boil, hence the pressure is maintained at normal level to prevent leak out and 
accidents. Further, temperature stability is desired for coolant. In this context, different parameter affecting the heat transfer must be studied carefully for proper and optimal performance of engineering system. Ostrach (1952) presented the similarity solution of natural convection along vertical isothermal plate. Kay (1966) reported that thermal conductivity of liquids with low Prandtl number varies linearly with temperature in range of $0^{\circ} \mathrm{F}$ to $400^{\circ} \mathrm{F}$. Arunachalam and Rajappa (1978) considered forced convection flow of liquid metals (having low Prandtl number) with variable thermal conductivity and derived explicit closed form of analytical solution. Carey and Mollendorf (1978) studied the effect of temperature dependent viscosity on free convective fluid flow. Crepeau and Clarksean (1997) discussed similarity solution of natural convection with internal heat generation which decays exponentially. Chaim (1998) studied heat transfer in fluid flow of low Prandtl number with variable thermal conductivity. Chamkha and Khaled (2001) obtained similarity solution of natural convection on an inclined plate with internal heat generation / absorption in presence of transverse magnetic field. Seddeek and Salem (2005) discussed the effect of variable viscosity and thermal diffusivity on mixed convection flow along vertical stretching sheet.

It is known that physical properties e.g. viscosity, thermal conductivity of fluids change with temperature. In most of the studies concerned with natural convection, generally, the simultaneous effect of temperature dependent viscosity and thermal conductivity occur. When these effects are included, the flow and heat transfer characteristic may change considerably. Hence, aim of the paper is to investigate the effects of varying viscosity and thermal conductivity on free convection flow of a viscous incompressible fluid and heat transfer along an isothermal vertical plate in the presence of heat sink.

\section{Formulation of the Problem}

Consider steady laminar two-dimensional free convection flow of a viscous incompressible fluid along a vertical plate kept at constant temperature $T_{w}$, in the presence of heat sink $Q$. The $x$-axis is taken along the plate and $y$-axis is normal to the plate. Incorporating the Buossinesq approximation within the boundary layer, the governing equations of continuity, momentum and energy [Schlichting (1968), Bansal (1977)], respectively are given by

$$
\frac{\partial u}{\partial x}+\frac{\partial v}{\partial y}=0 \text {, }
$$

$$
\begin{aligned}
& u \frac{\partial u}{\partial x}+v \frac{\partial u}{\partial y}=\frac{1}{\rho} \frac{\partial}{\partial y}\left(\mu^{*} \frac{\partial u}{\partial y}\right)+g \beta\left(T-T_{\infty}\right), \\
& \rho C_{p}\left(u \frac{\partial T}{\partial x}+v \frac{\partial T}{\partial y}\right)=\frac{\partial}{\partial y}\left(\kappa^{*} \frac{\partial T}{\partial y}\right)+Q .
\end{aligned}
$$

The boundary conditions are

$$
\begin{aligned}
& y=0: u=0, v=0, T=T_{w} \\
& y \rightarrow \infty: u=0, T=T_{\infty} .
\end{aligned}
$$

The variable viscosity [Carey and Mollendorf (1978)] and thermal conductivity [Kay (1966), Seddeek and Salem (2005)] are considered to vary linearly with temperature as given below, respectively

$$
\begin{aligned}
& \mu^{*}=\mu[1+\gamma(\theta-1 / 2)], \\
& \kappa^{*}=\kappa[1+\varepsilon \theta] .
\end{aligned}
$$

$\gamma$ and $\varepsilon$ are fluid characteristic and measure of the steepness of relation between the viscosity and thermal conductivity with temperature.

\section{Method OF SOLUTION}

Introducing the stream function $\psi(x, y)$ such that

$u=\frac{\partial \psi}{\partial y}$ and $v=-\frac{\partial \psi}{\partial x}$,

where $\psi(x, y)=4 v f(\eta)\left(\frac{G r}{4}\right)^{1 / 4}$ and the similarity

variable $\eta=\frac{y}{x}\left(\frac{G r}{4}\right)^{1 / 4}$.

Following Crepeau and Clarksean (1997), the heat sink is taken as given below

$$
Q=S\left\{\kappa\left(\frac{T_{w}-T_{\infty}}{x^{2}}\right)\left(\frac{G r}{4}\right)^{1 / 2}\left(T-T_{\infty}\right)\right\},
$$

where $G r$ is the Grashof number and $S(<0)$ is heat sink parameter.

Substituting equations (8) and (9) into the equations (2) and (3), along with the equations (5) and (6), the resulting non-linear ordinary differential equations are

$$
\{1+\gamma(\theta-1 / 2)\} f^{\prime \prime \prime}+\gamma \theta^{\prime} f^{\prime \prime}-2 f^{\prime 2}+3 f f^{\prime \prime}+\theta=0,
$$

and

$(1+\varepsilon \theta) \theta^{\prime \prime}+\varepsilon \theta^{\prime 2}+3 \operatorname{Pr} \theta^{\prime} f+S \theta=0$.

It is observed that the equation (1) is identically satisfied by $\psi(x, y)$. The boundary conditions are reduced to

$$
f(0)=0, f^{\prime}(0)=0, f^{\prime}(\infty)=0, \theta(0)=1 \text { and } \theta(\infty)=0 .
$$

The governing boundary layer equations (10) and (11) are coupled non-linear differential equations and 
solved using Runge-Kutta fourth order technique alongwith double shooting technique [Conte and Boor(1981)]. First of all, the higher order non-linear coupled differential equations (10) and (11) are decomposed into system of first order differential equations as given below

$$
\begin{aligned}
& \frac{\partial f}{\partial \eta}=U=f_{1}(\eta, f, U, V, \theta, W), \quad f(0)=0, \\
& \frac{\partial U}{\partial \eta}=V=f_{2}(\eta, f, U, V, \theta, W), \quad U(0)=0, \\
& \frac{\partial V}{\partial \eta}=f_{3}(\eta, f, U, V, \theta, W)= \\
& \left(-\gamma W V+2 U^{2}-3 f V-\theta\right) /\{1+\gamma(\theta-1 / 2)), \quad V(0)=? \\
& \frac{\partial \theta}{\partial \eta}=W=f_{4}(\eta, f, U, V, \theta, W), \quad \theta(0)=1, \\
& \frac{\partial W}{\partial \eta}=f_{5}(\eta, f, U, V, \theta, W)= \\
& {\left[-\varepsilon W^{2}-3 \operatorname{Pr} W f-S \theta\right] /(1+\varepsilon \theta), \quad W(0)=?}
\end{aligned}
$$

Now with the help of shooting technique $V(0)$ and $W(0)$ are approximated, as explained by Conte and Boor (1981). Hence the system of equations (13) to (17) is reduced to a system of initial value problem which is solved using Runge-Kutta technique. While shooting, to get the value of $V(0)$ and $W(0)$, care has been taken to shoot in steps and the shoots are improved in stages. While solving the system of equations, the step size is kept 0.005 .

\section{SKIN-FRICTION COEFFICIENT}

Skin-friction coefficient at the plate is given by

$C_{f}=2(1+\gamma / 2)(G r)^{-1 / 4} f^{\prime \prime}(0)$.

\section{NUSSELT NUMBER}

The rate of heat transfer in terms of the Nusselt number at the plate is given by

$$
N u=-(1+\varepsilon)(G r)^{-1 / 4} \theta^{\prime}(0) \text {. }
$$

\section{Particular Cases}

1. In the absence of heat sink and for constant thermal conductivity i.e. $S=0, \varepsilon=0$; the results of present paper are reduced to those obtained by Carey and Mollendorf (1978) and Hossain et.al. (2001).

2. In the absence of heat sink and constant viscosity and thermal conductivity i.e $S=0, \gamma=0$ and $\varepsilon=$ 0 ; the results of present paper are reduced to those obtained by Crepeau and Clarksean (1997) and Chamkha and Khaled (2001).

\section{RESUltS AND Discussion}

It is observed from Table 1 that the numerical values of $f^{\prime \prime}(0)$ and $\theta^{\prime}(0)$ for $\varepsilon=0.0$ and $S=0$ obtained in the present paper are in good agreement with those obtained by Hossain et.al (2001) and Cary \& Mollendorf (1978).

It is seen from Table 2 that the numerical results of $\theta^{\prime}(0)$ of present paper are in good agreement with those obtained by Chamkha et.al (2001) and Crepeau \& Claksean (1997) when $\gamma=0.0, \varepsilon=0.0$ and $S=0.0$.

Table 3 represents the values of $f^{\prime \prime}(0)$ and $-\theta^{\prime}(0)$ for different values of $\operatorname{Pr}$ and $S$ as these are required for the evaluation of skin-friction and Nusselt number. It is seen that with the increase in the Prandtl number the skin-friction decreases, while the rate of heat transfer increases. The decrease in heat sink parameter S causes decrease in the skin-friction and increase in the rate of heat transfer. With the increase in the value of $\varepsilon$, skin-friction increases, while rate of heat transfer decreases. On the contrary, as the value $\gamma$ increases skin-friction and rate of heat transfer decreases irrespective of the value of $S$.

Figure 1 represents that with the increase in the value Pr the velocity profiles decrease considerably when $0.1 \leq \operatorname{Pr} \leq 1.0$. Also it can be observed that boundary layer thickness also decreases considerably with increase in Prandtl number. It is interesting to note that the maximum of velocity profiles is achieved at almost same value of $\eta$. The temperature profiles and thermal boundary layer thickness decrease with the increase in Prandtl number which is seen from Fig. 2. It is observed from Figs. 3 and 4 that both velocity and temperature profiles decrease with the decrease in heat sink parameter $S$. Both the boundary layer and thermal boundary thicknesses decrease with decrease in heat sink parameter $S$.

Figures 5 and 6 show that variation of thermal conductivity affects velocity and temperature profiles. With the increase in the value of $\varepsilon$, the velocity and temperature of fluid increase, hence the variation of thermal conductivity cannot be neglected in case of low Prandtl number. From Figs. 7 and 8, effects of variation in viscosity on the velocity of fluid is seen near the plate, while negligible effect is noted on the temperature of fluid. In the case of lower values of $\gamma$, the velocity profiles are steeper and on temperature profiles the effects are negligible. 
N.C. Mahanti and P. Gaur / JAFM, Vol. 2, No. 1, pp. 23-28, 2009.

\section{Conclusions}

1. The velocity and the temperature of the fluid decrease with the increase in Prandtl number.

2. The velocity and thermal boundary layers thickness decrease with increase in Prandtl number.

3. The velocity and the temperature of the fluid decrease with the decrease in heat sink parameter $S$.

4. The velocity and thermal boundary layers thickness decrease with decrease in heat sink parameter $S$.

5. The increase in thermal conductivity parameter $\varepsilon$ increases the velocity and temperature of fluid irrespective of value of heat sink parameter $S$.

6. The increase in viscosity parameter $\gamma$ decreases the velocity of fluid near the plate however the effect of viscosity parameter $\gamma$ is negligible on the temperature of the fluid.

7. Skin-friction coefficient increases, while rate of heat transfer decreases with the increase in Prandtl number.

8. Skin-friction coefficient decreases, while rate of heat transfer increases with the decrease in the heat sink parameter $S$.

9. Skin-friction coefficient increases, while rate of heat transfer decreases with the increase in thermal conductivity parameter $\varepsilon$.

10. Skin-friction increases with the increase in viscosity parameter $\gamma$ and the effects are negligible in the case of rate of heat transfer.

\section{REFERENCES}

Arunachalam, M. and N.R. Rajappa (1978). Forced convection in liquid metals with variable thermal conductivity and capacity. Acta Mechanica 31, 25-31.

Bansal, J.L. (1977). Viscous Fluid Dynamics, Oxford \& IBH Pub. Co., New Delhi, India.
Carey, V.P. and J.C. Mollendorf (1978). Natural convection in liquid with temperature dependent viscosity. Proc. $6^{\text {th }}$ International Heat Transfer Conference, Toronto, 2, 211-217.

Chaim, T.C. (1998). Heat transfer in a fluid with variable thermal conductivity over stretching sheet. Acta Mechanica 129, 63-72.

Chamkha, A.J. and A.R.A. Khaled (2001). Similarity solutions for hydromagnetic simultaneous heat and mass transfer by natural convection from an inclined plate with internal heat generation or absorption. Heat and Mass Transfer 37, 117-123.

Conte, S.D. and C. Boor (1981). Elementary Numerical Analysis, McGraw-Hill Book Co., New York.

Crepeau, J.C. and R. Clarksean (1997). Similarity solution of natural convection with internal heat generation. ASME J. of Heat Transfer 119, 183-185.

Ostrach, S. (1952). An analysis of laminar free convective flow and heat transfer about a flat plate parallel to direction of the generating body force. NACA Technical Report 1111.

Kay, W.M. (1966). Convective Heat and Mass Transfer. McGraw-Hill Book Co., New York.

Seddeek, M.A. and A.M. Salem (2005). Laminar mixed convection adjacent to vertical continuously stretching sheet with variable viscosity and variable thermal diffusivity. Heat and Mass Transfer 41, 1048-1055.

Schlichting, H. (1968). Boundary Layer Theory, McGrawHill Book Co., New York.

Table 1 Values of $f^{\prime \prime}(0)$ and $\theta^{\prime}(0)$ for different values of $\gamma$ when $\operatorname{Pr}=1.0$ and $S=0$ are compared with the results obtained by Hossain et.al (2001) and Carey \& Mollendorf (1978).

\begin{tabular}{|c|c|c|c|c|c|c|}
\hline \multirow{2}{*}{$\gamma$} & \multicolumn{2}{|c|}{ Hossain et.al (2001) } & \multicolumn{2}{c|}{ Carey \& Mollendorf (1978) } & \multicolumn{2}{c|}{ Present paper } \\
\cline { 2 - 7 } & $f^{\prime \prime}(0)$ & $\theta^{\prime}(0)$ & $f^{\prime \prime}(0)$ & $\theta^{\prime}(0)$ & $f^{\prime \prime}(0)$ & $\theta^{\prime}(0)$ \\
\hline 0.0 & 0.6421 & -0.5671 & 0.6422 & -0.5671 & 0.642187 & -0.567145 \\
\hline 0.8 & 0.5050 & -0.5469 & 0.5050 & -0.5469 & 0.505014 & -0.546940 \\
\hline 1.6 & 0.4222 & -0.5281 & 0.4233 & -0.5315 & 0.422341 & -0.531531 \\
\hline-1.6 & 2.0411 & -0.6514 & 2.0416 & -0.6514 & 2.041617 & -0.651368 \\
\hline
\end{tabular}


N.C. Mahanti and P. Gaur / JAFM, Vol. 2, No. 1, pp. 23-28, 2009.

Table 2 Values of $\theta^{\prime}(0)$ for different values of Pr are compared with the results obtained by Chamkha et.al (2001) and Crepeau \& Clarksean (1997) when $S=0.0$.

\begin{tabular}{|c|c|c|c|}
\hline \multirow{2}{*}{$\operatorname{Pr}$} & Chamkha et.al (2001) & Crepeau \& Clarksean (1997) & Present paper \\
\cline { 2 - 4 } & $\theta^{\prime}(0)$ & $\theta^{\prime}(0)$ & $\theta^{\prime}(0)$ \\
\hline 0.1 & -0.2119 & -02302 & -0.230136 \\
\hline 1.0 & -0.5646 & -0.5671 & -0.567145 \\
\hline 10 & -1.1720 & -1.169 & -1.166270 \\
\hline
\end{tabular}

Table 3 Values of $f^{\prime \prime}(0)$ and $-\theta^{\prime}(0)$ for different values of $\operatorname{Pr}$ and $S$.

\begin{tabular}{|c|c|c|c|c|c|c|}
\hline & \multicolumn{2}{|l|}{$\gamma=-0.4$} & \multicolumn{2}{|l|}{$\gamma=0.0$} & \multicolumn{2}{|l|}{$\gamma=0.4$} \\
\hline $\begin{array}{l}S=0.0 \\
\operatorname{Pr}=0.01\end{array}$ & $f^{\prime \prime}(0)$ & $-\theta^{\prime}(0)$ & $f^{\prime \prime}(0)$ & $-\theta^{\prime}(0)$ & $f^{\prime \prime}(0)$ & $-\theta^{\prime}(0)$ \\
\hline$\varepsilon=0.0$ & 1.118735 & 0.081007 & 0.987754 & 0.080592 & 0.8918468 & 0.080222 \\
\hline$\varepsilon=0.1$ & 1.221255 & 0.076171 & 0.9913039 & 0.075793 & 0.895466 & 0.075456 \\
\hline$\varepsilon=0.3$ & 1.127786 & 0.068516 & 0.997233 & 0.068196 & 0.901519 & 0.07910 \\
\hline $\begin{array}{l}S=0.0 \\
\operatorname{Pr}=0.023\end{array}$ & & & & & & \\
\hline$\varepsilon=0.0$ & 1.083838 & 0.120014 & 0.952202 & 0.119131 & 0.856223 & 0.118351 \\
\hline$\varepsilon=0.1$ & 1.088379 & 0.112941 & 0.956866 & 0.112134 & 0.860912 & 0.111420 \\
\hline$\varepsilon=0.3$ & 1.096049 & 0.101735 & 0.964749 & 0.101046 & 0.868843 & 0.100435 \\
\hline $\begin{array}{l}S=-0.1 \\
\operatorname{Pr}=0.023\end{array}$ & & & & & & \\
\hline$\varepsilon=0.0$ & 0.960866 & 0.332112 & 0.828767 & 0.331572 & 0.733861 & 0.331110 \\
\hline$\varepsilon=0.1$ & 0.969182 & 0.311975 & 0.836995 & 0.311474 & 0.741909 & 0.311044 \\
\hline$\varepsilon=0.3$ & 0.983516 & 0.280223 & 0.851179 & 0.279784 & 0.755798 & 0.279407 \\
\hline $\begin{array}{l}S=-0.2 \\
\operatorname{Pr}=0.023\end{array}$ & & & & & & \\
\hline$\varepsilon=0.0$ & 0.898653 & 0.457014 & 0.768852 & 0.456592 & 0.676233 & 0.456237 \\
\hline$\varepsilon=0.1$ & 0.908308 & 0.429227 & 0.778209 & 0.428831 & 0.676824 & 0.427979 \\
\hline$\varepsilon=0.3$ & 0.925107 & 0.385430 & 0.794511 & 385078 & 0.700987 & 0.384781 \\
\hline
\end{tabular}

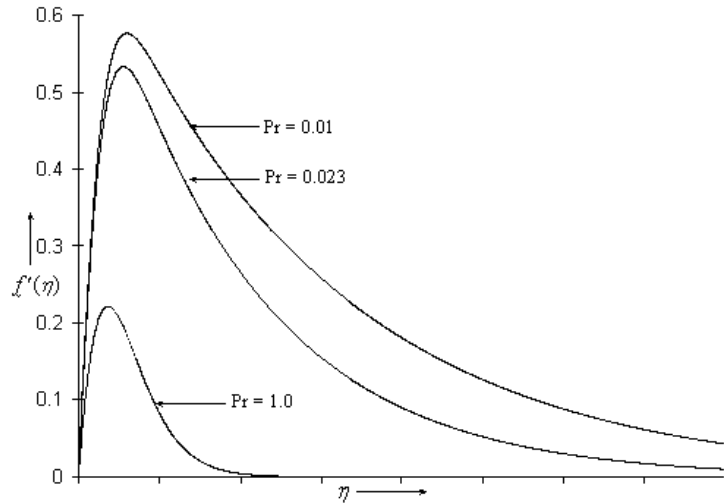

Fig. 1. Velocity distribution versus $\eta$ when $\varepsilon=0.0$, $\gamma=0.0$ and $S=0.0$.

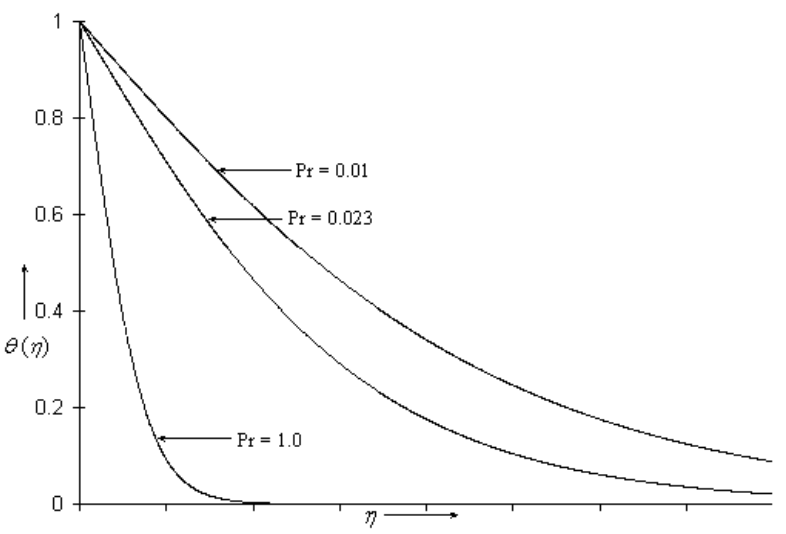

Fig. 2. Temperature distribution versus $\eta$ when $\varepsilon=0.0$, $\gamma=0.0$ and $S=0.0$. 
N.C. Mahanti and P. Gaur / JAFM, Vol. 2, No. 1, pp. 23-28, 2009.
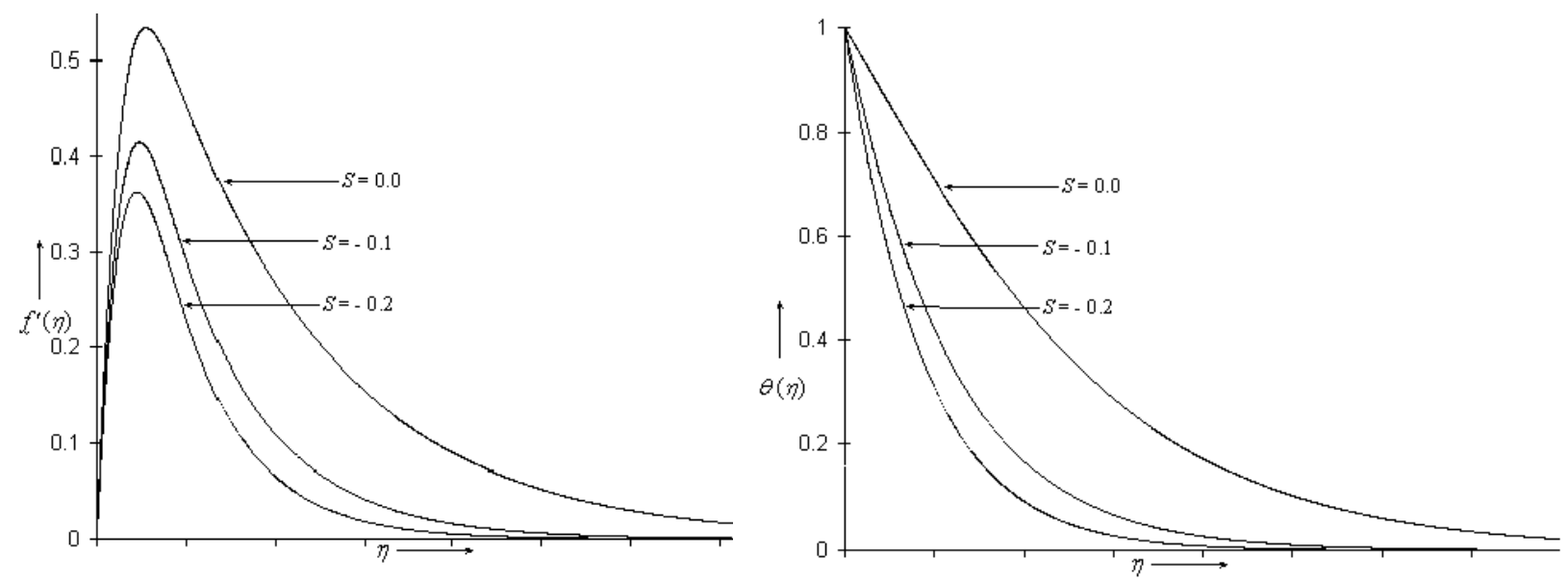

Fig. 3. Velocity distribution versus $\eta$ when $\varepsilon=0.0$, $\gamma=0.0$ and $\operatorname{Pr}=0.023$.

Fig. 4. Temperature distribution versus $\eta$ when $\varepsilon=0.0$, $\gamma=0.0$ and $\operatorname{Pr}=0.023$.
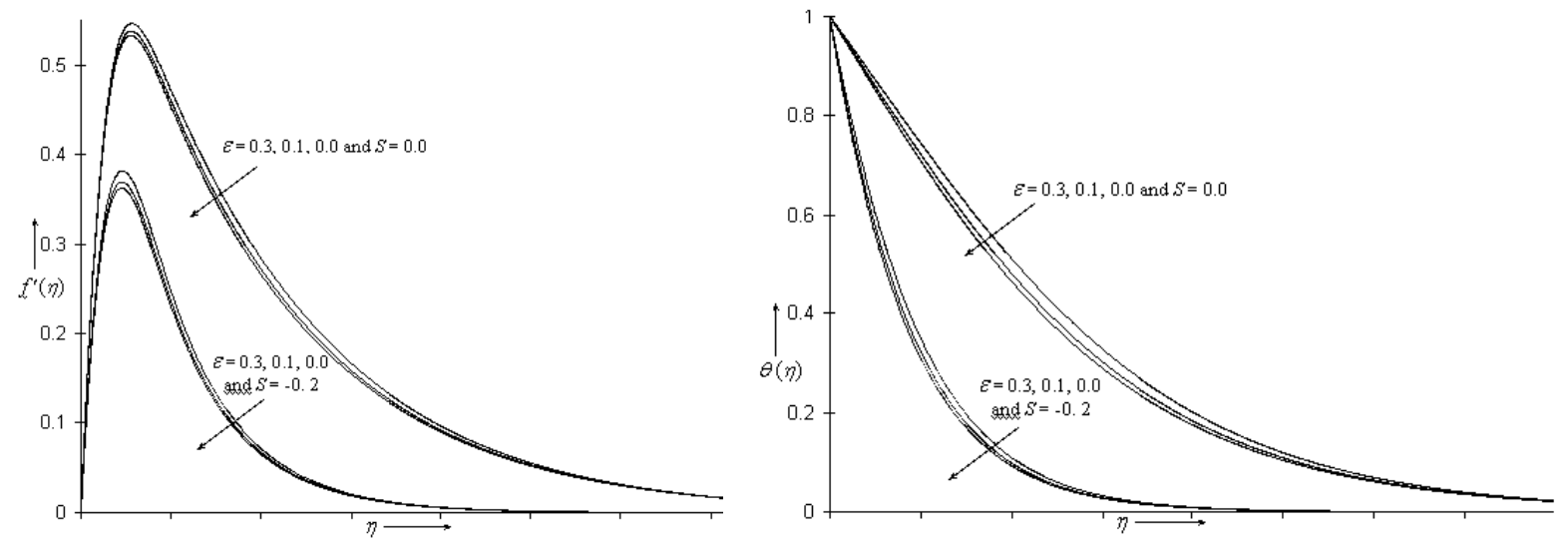

Fig. 5. Velocity distribution versus $\eta$ when $\gamma=0.0$ and $\operatorname{Pr}=0.023$.

Fig. 6. Temperature distribution versus $\eta$ when $\gamma=0.0$ and $\operatorname{Pr}=0.023$.
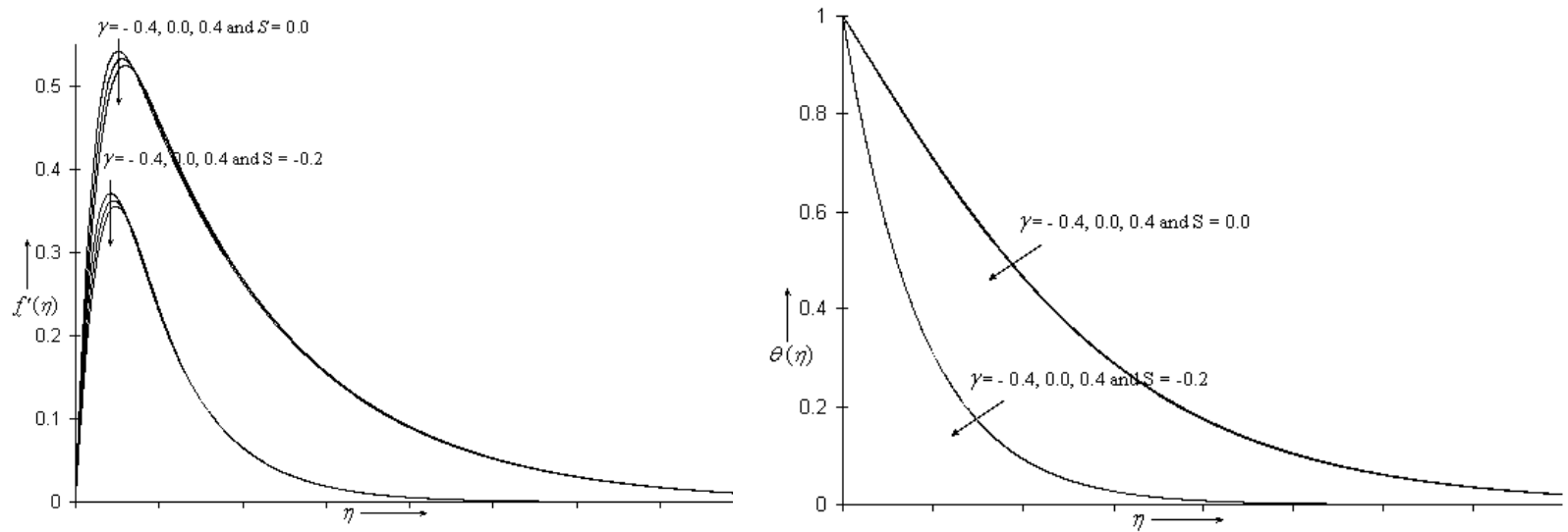

Fig. 7. Velocity distribution versus $\eta$ when $\varepsilon=0.0$ and $\operatorname{Pr}=0.023$.

Fig. 8. Temperature distribution versus $\eta$ when $\varepsilon=0.0$ and $\operatorname{Pr}=0.023$. 